\title{
EFFECTIVE LABORATORY QUALITY MANAGEMENT TOWARDS PREVENTING MEDICAL LIABILITY CASES
}

\author{
A S Kanagasabapathy* \\ Principal Consultant, Quality Council of India (Hon)
}

The last decade has seen a rapid expansion of the various branches of health care services in India. This has seen a corresponding increase in consumer knowledge, which has resulted in (a) increased demand for high quality services and (b) these services to be provided in a timely manner. Medical laboratory plays a central role in the delivery of these services as over $70 \%$ of clinical decisions are taken based on laboratory reports (1).

An important component in the whole process is laboratory error and an expectation to get error free services. With growing awareness amongst customers, in this case largely patients, and increasing cost, medico- legal litigations are on the rise. Court cases are often filed against doctors by patients for real or perceived reasons of dissatisfaction. Medical malpractice law suits against medical laboratories are also on the rise. This has placed a significant onus and urgency on clinical laboratories to ensure that quality and error free work practices are maintained.

There has been a steady improvement in the quality of tests due to improved technology. Laboratory automation has also taken on a new level of importance in improving quality. We have the ability now to get instruments interfaced to various laboratory information systems. Information technology has consequently taken a giant leap in the field of biomedical equipment to drastically reduce manual intervention during the analytical procedure without compromising on established levels of care.

Despite the above developments, errors due to pre-analytical factors continue to occur and we have to focus on these

\section{Address for Correspondence :}

\section{Dr A S Kanagasabapathy}

Consultant, Quality Council of India (Hon)

Former Professor \& Head of Clinical Biochemistry,

CMC Vellore $\cdot$ Mobile : +91-9704335533

E-mail : askanag@gmail.com issues to reduce the errors. The high frequency of errors still attributable to processes external to the laboratory requires special attention and efforts to prevent such errors. This will ultimately contribute to prevention of direct or indirect involvement of the laboratory in medical liability cases.

In the laboratory, mistakes are liable to occur in each possible step starting from preparation and sample collection down to actual dispatch of the report. For example, consider the following scenarios:

(a) A blood sample was drawn from a patient for electrolyte estimation but was wrongly collected into a tube containing anticoagulant with $\mathrm{K}+($ EDTA). On realizing his error the technician tips the sample into the correct tube. As a result of this the measured $\mathrm{K}+$ value in this sample is falsely high due to the added $K+$ in the EDTA while the $\mathrm{Ca}$ is falsely low due as the available $\mathrm{Ca}$ in the sample got chelated by the EDTA. All of this is due to a simple mistake of collecting the sample in the wrong container!

(b) A hospitalized diabetic patient whose blood glucose had been brought down to and maintained at euglycaemic levels was on a particular day found to be quite abnormal. A thorough enquiry revealed that the duty doctor forgot to enter the order for insulin injection for the next morning, which was consequently not administered by the nurse. Hence, the abnormal blood glucose value.

(c) Medico legal litigations involving FNAC are a result of a poorly prepared sample. This fact is neither mentioned in the report nor reflected in the diagnostic conclusion. As a result, the clinician assumes that conditions for a reliable diagnosis were met and on this basis plans a therapeutic procedure (2). 
(d) Cytological diagnosis has its own fallacies. For example, the sensitivity of FNAC in breast lesions is $90-95 \%$. Failure to communicate the limitations of FNAC in various lesions can result in surgeries, which may be unwarranted. These form a bulk of medico legal litigations involving practice of cytology (3).

There are innumerable examples such as these that can be quoted, which are certainly a timely reminder to clinical laboratories to make sure that pre-analytical steps are very important in the provision of valid results and clinical management. As consumers raise the bar on their expectation of provider quality, laboratories must respond by using management methods of continuous improvement to reduce and eliminate any and all sources of errors and defects in their work flow, analytical activities and customer service execution.

Yet another factor to consider is how the senior laboratory personnel can provide value adding to the results generated by giving interpretative comments on laboratory reports. Vasikaran has recently published an interesting article on behalf of the APFCB (4) on the quality of interpretative commenting on common clinical chemistry results. While most of the anatomical pathology reports carry interpretative comments, clinical pathology/clinical biochemistry reports mostly do not. However, clinicians appreciate interpretative comments on the more complex tests and feel that they can help or influence in patient management. This can potentially save time and improve the diagnostic process. 31 senior laboratory personnel (27 from Asia - Pacific region and 4 from Africa) participated in this survey, out of which 11 were from India. The quality of the comments returned by participants was diverse and some reflected incorrect or misleading interpretation and advice. While interpretative commenting is an important laboratory activity, the results of this study suggest that there is room for improvement in the quality of interpretative comments.

In this regard, several legal steps have been taken by the Government of India to help the public get maximum compensation for errors made by doctors and laboratories. The two important Acts need a special mention:

(1) The Consumer Protection Act 1986 which was enacted in order to provide speedy and inexpensive redress of consumers' grievances

(2) The Right to Information Act 2005 (RTI Act) which was enacted to provide access to records of the Central Government and State Governments.
Medico-legal disputes involving laboratories are only in the infant stage in several of the Asia - Pacific region (Personal Communication - Joseph Lopez, Leslie Lai and Chris Lam). In Australia, law against malpractices by doctors protects consumers and this would apply to medical laboratories as well (Personal Communication - Samuel Vasikaran).

In Malaysia, the most notable and frequent instances when the medical laboratory is mentioned in court are for cases involving drugs of abuse. Since all government hospital laboratories are designated drug detection centres, clinical biochemists are sometimes called to testify in court on the results they produce (Personal Communication - Joseph Lopez). The Malaysian Medical Council has issued guidelines and directives with specific reference to medical records and medical reports to safeguard the patient and members of the public to ensure propriety in professional practice and to prevent abuse of professional privileges (5).

\section{How to avoid laboratory errors towards preventing involvement of the laboratory in medical liability cases?}

1. The laboratory and hospital need to design systems that reduce the possibility of errors and to rapidly identify and resolve the errors that do occur. Because the pre- and post-analytical processes extend into the clinical operations of the hospital, the laboratory can play an important role in promoting patient safety by assisting clinicians with test ordering, communicating test results appropriately and aiding in the interpretation of results.

2. For patients, some of the most devastating medical mistakes can start in the laboratory, where studies show that 3 percent to 5 percent of the billions of specimens taken each year are defective, be it a biopsy that does not extract the tumor cells, blood that is not drawn correctly or a mix-up with another patient's sample (6). Therefore sampling must be made with proper care.

3. The laboratories should incorporate effective specimen rejection criteria towards preventing the process of defective/doubtful samples.

4. Specimen integrity has definitely increased with the introduction of bar-coded Technology. When the system is used properly, we know with certainty the patient to whom a specimen belongs. The laboratory should avoid critical specimen errors such as incorrect patient ID, incorrectly labeled specimen, incorrect time on label, 
delayed transportation to laboratory, computer-related error, insufficient quantity, hemolysis, etc (7).

5. Bidirectional interface and on-line data transfer: Interface of machines will help in increasing turn around times, less error in patient samples through positive identification, easily carrying out management stats, ease of transmitting results to digital medical records thus increasing paperless environment, less error in transmitting results to reports, better maintenance of results quality through error reduction.

6. Reporting of patient's test results at the earliest possible time is of paramount importance. Laboratories have been penalized by the courts in our country for the exorbitant delays in giving crucial test reports

7. Total compliance with ISO 15189:2007, with special emphasis on documentation.

It is the foremost responsibility of the laboratory to make sure that patients always receive the service they deserve - a good quality result that ensures our referring clinicians can practice good medicine. Patients deserve quality. As for any other type of medical errors, the most effective path to improvement in laboratory performance is the implementation of a total quality management system, encompassing a multifaceted strategy for process and risk analysis, based on error detection, prevention and management. Let us remember that a solid base for quality is to establish a strong foundation of Quality Assurance and the watchwords are "SAY WHAT YOU DO, DO WHAT YOU SAY \& PROVE IT”.
*Author is a member Editorial Board IJCB and Former Professor \& Head of Clinical Biochemistry, CMC Vellore, established the ACBI EQA programme at CMC Vellore as early as 1978 . He is associated with the $W H O$ in various laboratory quality control activities and has been the author/coauthor of 3 WHO documents. In 1997 he was the Chairman of Technical Committee constituted by the NABL for formulating specific guidelines criteria for medical laboratories. He has contributed significantly towards the preparation of NABL document \# 112 of 2005. He is presently associated with Quality Council of India in QCl's valuable venture of promoting standards of performance towards accreditation of the State government hospital laboratories.

\section{REFERENCES}

1. Dighe MS, Markar RS, Lewandrowski KB. Medicolegal liability in laboratory medicine. Semin Diagn Pathol 2007; 24 (2): 98-107.

2. Kocjan G. Fine needle aspiration cytology, "Diagnostic principles and dilemmas" $1^{\text {st }}$ edition, 2006 (Chapter 2); 11-13.

3. Orell SR, Sterrett GF, Max N-I Walters, Whitaker D. Manual and Atlas of Fine needle aspiration cytology, $3^{\text {rd }}$ edition, 2006 (Chapter 7); 150-51.

4. Vasikaran SD, Lai LC, Sethi S, Lopez JB, Sikaris KA. Quality interpretative commenting on common clinical chemistry results in Asia - Pacific region and Africa. Clin Chem Lab Med 2009; 47(8): 963-70.

5. Guidelines of the Malaysian Medical Council MMC Guideline 002 / 2006.

6. Landro L. Hospitals move to cut dangerous lab errors. The Wall Street Journal. 2006; June 14.

7. Bologna LJ, Motter M. Life after phlebotomy development: Reducing major patient and specimen identification errors. J Healthcare Information Management 2002; 16(1): 65-70. 